TRANSACTIONS OF THE

AMERICAN MATHEMATICAL SOCIETY

Volume 363, Number 1, January 2011, Pages 131-142

S 0002-9947(2010)04888-3

Article electronically published on August 24, 2010

\title{
UNIQUENESS OF HOLOMORPHIC CURVES INTO ABELIAN VARIETIES
}

\author{
MATTHEW DULOCK AND MIN RU
}

\begin{abstract}
In this paper, we first give a slight improvement of Yamanoi's truncated second main theorem for holomorphic maps into abelian varieties. We then use the result to study the uniqueness problem for such maps. The results obtained generalize and improve E. M. Schmid's uniqueness theorem for holomorphic maps into elliptic curves. In the last section, we consider algebraic dependence for a finite collection of holomorphic curves into an abelian variety.
\end{abstract}

\section{INTRODUCTION}

A well-known result of Nevanlinna states that if $f, g: \mathbb{C} \rightarrow \mathbb{P}^{1}(\mathbb{C})$ are nonconstant holomorphic maps such that $f^{-1}\left\{a_{i}\right\}=g^{-1}\left\{a_{i}\right\}$ for distinct $a_{1}, \cdots, a_{5} \in$ $\mathbb{P}^{1}(\mathbb{C})$, then $f \equiv g$. The problem of generalizing this result to various classes of complex manifolds has attracted much attention. H. Fujimoto studied the uniqueness of meromorphic mappings from $\mathbb{C}^{n}$ into $\mathbb{P}^{m}(\mathbb{C})$ (see, for example, $[\mathrm{Fu}]$ ). E.M. Schmid, in $[\mathbf{S c}$, considered the questions of this type for holomorphic maps from $\mathbb{C}$ into elliptic curves and obtained the following result: If $E$ is an elliptic curve, and if $f, g: \mathbb{C} \rightarrow E$ are non-constant holomorphic mappings such that $f^{-1}\left\{a_{i}\right\}=g^{-1}\left\{a_{i}\right\}$ as point sets for distinct $a_{1}, \cdots, a_{5} \in E$, then $f \equiv g$ on $\mathbb{C}$.

In each case of dealing with the uniqueness problem, an inequality of truncated second main theorem type was used to prove the result. Recently, K. Yamanoi established a truncated second main theorem for holomorphic maps into an abelian variety $A$ (see [Ya]), and Noguchi, Winklemann, and Yamanoi extended the result to the semi-abelian variety $A([\mathrm{No}-\mathrm{W}-\mathrm{Y}])$. In this paper, we first give an improvement of Yamanoi's result by considering only the points with multiplicity 1 . As a consequence, we show that if a holomorphic curve $f: \mathbb{C} \rightarrow A$ is totally (or completely) ramified over an effective reduced ample divisor on $A$, where $A$ is an abelian variety, then $f$ is not Zariski dense. In particular, if $f: \mathbb{C} \rightarrow E$ is holomorphic, where $E$ is an elliptic curve, and $f$ is totally (or completely) ramified over a point in $E$, then $f$ must be constant. We then use the improved second main theorem to obtain uniqueness results for holomorphic maps from $\mathbb{C}$ into an abelian variety $A$. In particular, we extend and improve E.M. Schmid's result. In the last section, we prove an algebraic dependence result for a finite collection of holomorphic curves into an abelian variety.

Received by the editors July 3, 2007 and, in revised form, July 14, 2008.

2000 Mathematics Subject Classification. Primary 32H30; Secondary 14K20.

Key words and phrases. Abelian variety, uniqueness theorem, holomorphic mappings, algebraic dependency, Nevanlinna theory.

The second author was supported in part by NSA under grant number H98230-09-1-0004.

(C)2010 American Mathematical Society

Reverts to public domain 28 years from publication 


\section{An IMPROVEMENT OF YAMANOI'S TRUNCATED SECOND MAIN THEOREM}

Definition 2.1. Let $V$ be a complex vector space of dimension $n$ and let $\Lambda$ be a lattice of rank $2 n$. The quotient space $A=V / \Lambda$ is called an $n$-dimensional complex torus. $A$ is an abelian variety if it can be embedded into a projective space.

If $A$ is a complex torus and $\operatorname{dim} A=1$, then we can identify $H^{2}(A, \mathbb{Z})$ with $\mathbb{Z}$. So, if $a \in A$ and $L$ is the line bundle determined by $a$, then $c_{1}(L)=1$. Therefore, $L$ is a positive holomorphic line bundle on $A$, and hence $A$ is an abelian variety. A one-dimensional abelian variety is called an elliptic curve.

Suppose $A$ is an abelian variety, $\omega$ a smooth $(1,1)$-form on $A$, and $f: \mathbb{C} \rightarrow A$ is holomorphic. We define, for $r>1$, the characteristic function of $f$ with respect to $\omega$ by

$$
T_{f}(r, \omega)=\int_{1}^{r} \frac{d t}{t} \int_{|z|<t} f^{*} \omega
$$

For a holomorphic line bundle $L$ over $A$, we define (up to a bounded term) $T_{f}(r, L)=$ $T_{f}\left(r, c_{1}(L)\right)$ where $c_{1}(L)$ is the Chern form of $L$ (with respect to some metric). We note that if $L$ is big, then $T_{f}(r, L) \rightarrow \infty$ as $r \rightarrow \infty$, and if $\left(-L_{1}\right) \otimes L_{2}$ is big, then $T_{f}\left(r, L_{1}\right) \leq T_{f}\left(r, L_{2}\right)$.

Let $E=\sum m_{\alpha} z_{\alpha}$ be an effective divisor on $\mathbb{C}$. We define the counting functions:

$$
\begin{gathered}
n(t, E)=\sum_{\left|z_{\alpha}\right|<t} m_{\alpha}, \\
N(r, E)=\int_{1}^{r} \frac{n(t, E)}{t} d t .
\end{gathered}
$$

We define the truncated counting functions:

$$
\begin{gathered}
\bar{n}(t, E)=\sum_{\left|z_{\alpha}\right|<t} \min \left\{1, m_{\alpha}\right\}, \\
\bar{N}(r, E)=\int_{1}^{r} \frac{\bar{n}(t, E)}{t} d t .
\end{gathered}
$$

For a discrete subset $S \subset \mathbb{C}$, we let $n(t, S)=\#(S \cap\{z:|z|<t\})$ and

$$
N(r, S)=\int_{1}^{r} \frac{n(t, S)}{t} d t
$$

Let $k \in \mathbb{N} \cup\{\infty\}$, and for an effective divisor $E=\sum m_{\alpha} z_{\alpha}$, we let

$$
S u p p_{\leq k} E=\bigcup_{0<m_{\alpha} \leq k}\left\{z_{\alpha}\right\}, E_{\leq k}=\sum_{m_{\alpha} \leq k} m_{\alpha} z_{\alpha}
$$

and

$$
\operatorname{Supp}_{>k} E=\bigcup_{m_{\alpha}>k}\left\{z_{\alpha}\right\}, E_{>k}=\sum_{m_{\alpha}>k} m_{\alpha} z_{\alpha} .
$$

Let $D$ be a divisor on $A$. We denote by $\mathcal{O}(D)$ the line bundle determined by $D$. We now improve Yamanoi's truncated second main theorem (see $\mathrm{Ya}$, or [No-W-Y]. For the second main theorem without truncation, see Siu-Yeung $[\underline{\mathrm{Si}-\mathrm{Y}]}]$ ). 
Theorem 2.2. Suppose that $A$ is an abelian variety, $D$ is a reduced effective divisor on $A$, and $F$ is an ample divisor. If $f: \mathbb{C} \rightarrow A$ is a Zariski dense holomorphic map, then for every $\epsilon>0$,

$$
\| T_{f}(r, \mathcal{O}(D)) \leq N\left(r, f^{*} D_{\leq 1}\right)+\epsilon T_{f}(r, F) .
$$

Here, $\|$ means that the inequality holds for all $r>1$, except possibly on a set of finite Lebesgue measure.

Proof. For every $\epsilon>0$, by Yamanoi's truncated second main theorem (see Ya]), we have

$$
\| T_{f}(r, \mathcal{O}(D)) \leq \bar{N}\left(r, f^{*} D\right)+\frac{\epsilon}{2} T_{f}(r, F) .
$$

Since

$$
\begin{gathered}
N\left(r, f^{*} D\right) \geq \bar{N}\left(r, f^{*} D_{\leq 1}\right)+2 \bar{N}\left(r, f^{*} D_{>1}\right)=2 \bar{N}\left(r, f^{*} D\right)-\bar{N}\left(r, f^{*} D_{\leq 1}\right), \\
\bar{N}\left(r, f^{*} D\right) \leq \frac{1}{2} \bar{N}\left(r, f^{*} D_{\leq 1}\right)+\frac{1}{2} N\left(r, f^{*} D\right)=\frac{1}{2} N\left(r, f^{*} D_{\leq 1}\right)+\frac{1}{2} N\left(r, f^{*} D\right) .
\end{gathered}
$$

Hence,

$$
\begin{aligned}
\| T_{f}(r, \mathcal{O}(D)) & \leq \frac{1}{2} N\left(r, f^{*} D_{\leq 1}\right)+\frac{1}{2} N\left(r, f^{*} D\right)+\frac{\epsilon}{2} T_{f}(r, F) \\
& \leq \frac{1}{2} N\left(r, f^{*} D_{\leq 1}\right)+\frac{1}{2} T_{f}(r, \mathcal{O}(D))+\frac{\epsilon}{2} T_{f}(r, F) .
\end{aligned}
$$

Thus,

$$
\| \frac{1}{2} T_{f}(r, \mathcal{O}(D)) \leq \frac{1}{2} N\left(r, f^{*} D_{\leq 1}\right)+\frac{\epsilon}{2} T_{f}(r, F) .
$$

This gives the desired inequality.

Corollary 2.3. Suppose that $A$ is an abelian variety and $f: \mathbb{C} \rightarrow A$ is holomorphic. Assume that there is a reduced effective ample divisor $D$ on $A$ such that

$$
\limsup _{r \rightarrow+\infty} \frac{N\left(r, f^{*} D_{\leq 1}\right)}{T_{f}(r, \mathcal{O}(D))}<1
$$

then $f$ is not Zariski dense in $A$. In particular, if there is an effective reduced ample divisor $D$ on $A$ such that $f$ is totally ramified over $D\left(i . e ., S u p p_{\leq 1} f^{*} D=\emptyset\right)$, then $f$ is not Zariski dense in $A$.

Corollary 2.4. Suppose that $E$ is an elliptic curve and $f: \mathbb{C} \rightarrow E$ is holomorphic. Assume that there is a point $a \in E$ such that $f$ is totally ramified over a (i.e., $\left.\operatorname{Supp}_{\leq 1} f^{*}\{a\}=\emptyset\right)$, then $f$ is constant.

\section{Some Results about abelian varieties}

Let $A$ be an abelian variety. A polarization on $A$ is the first Chern class $c_{1}(L)$ of a positive line bundle $L$ on $A$. By abuse of notation, we sometimes consider the positive line bundle $L$ itself as a polarization (note by the definition of abelian variety, we know there always is such a bundle). If we can choose a basis, $\lambda_{1}, \ldots, \lambda_{2 n}$, for $\Lambda$ over $\mathbb{Z}$ such that in terms of dual coordinates, $x_{1}, \ldots, x_{2 n}$, the Chern form $c_{1}(L)=\sum_{j=1}^{n} 1 d x_{j} \wedge d x_{n+j}$, then we say that $L$ is a principal polarization. Equivalently, $L$ is principally polarized if $\operatorname{dim} H^{0}(A, L)=1$. If $A$ is an elliptic curve and $L$ is the line bundle determined by $(a)$ for $a \in A$, then since $c_{1}(L)=1, L$ is principally polarized. 
For a holomorphic line bundle $L$ over a compact complex manifold $M$, it is well known that if $L$ is positive, then $L$ is ample; that is, $H^{0}(M, \mathcal{O}(k L))$ gives an embedding into a projective space for some $k>0$. The following result of Lefschetz states that in the case of abelian variety we can be more precise. We refer the reader to $\mathrm{Gr}-\mathrm{H}$ ] for the proof.

Theorem 3.1 (Lefschetz). Suppose $A$ is an abelian variety, and $L$ a positive line bundle over $A$. Then,

(1) $H^{0}(A, \mathcal{O}(2 L))$ has no base points (i.e., it defines a map into a projective space).

(2) If $k>2$, then $H^{0}(A, \mathcal{O}(k L))$ gives an embedding into a projective space.

To study the uniqueness of holomorphic curves into an abelian variety $A$, we will need a deeper understanding of the map defined in (1) of Theorem 3.1. More specifically, we wish to study the injectivity of this map.

We recall the Decomposition Theorem 4.3.1 on page 75 in $\mathrm{Bi}-\mathrm{L}$. Let $F_{1}+\cdots+F_{r}$ be the decomposition of the fixed part of $|L|$ and let $|M|$ be the moving part of $|L|$. Write $N_{j}=\mathcal{O}\left(F_{j}\right)$. Let $A_{M}=A / K(M)_{0}$, and $A_{N_{j}}=A / K\left(N_{j}\right)_{0}$, where $K(M)_{0}$ and $K\left(N_{j}\right)_{0}$ are the subtori of $A$ with respect to the line bundles $M$ and $N_{j}$ (see $\mathrm{Bi}-\mathrm{L}$ for the precise definition). Then Lemma 3.3.2 in Bi-L provides ample line bundles $\bar{M}$ on $A_{M}$ and $\bar{N}_{j}$ on $A_{N_{j}}$ with $M=p_{M}^{*} \bar{M}$ and $N_{j}=p_{j}^{*} \bar{N}_{j}$, where $p_{M}: A \rightarrow A_{M}:=A / K(M)_{0}$ and $p_{j}: A \rightarrow A_{N_{j}}:=A / K\left(N_{j}\right)_{0}$ are the natural projections. The pairs $\left(A_{M}, \bar{M}\right)$ and $\left(A_{N_{j}}, \bar{N}_{j}\right)$ are polarized abelian varieties. In particular, the $\bar{N}_{j}$ s define principal polarizations on the abelian variety $A_{N_{j}}$, since $\operatorname{dim} H^{0}\left(A_{N_{j}}, \bar{N}_{j}\right)=\operatorname{dim} H^{0}\left(A, N_{j}\right)=1$. The Decomposition Theorem 4.3.1 in Bi-L] states that

$p=\left(p_{M}, p_{1}, \ldots, p_{r}\right):(A, L) \rightarrow\left(A_{M} \times A_{N_{1}} \times \cdots \times A_{N_{r}}, q_{M}^{*} \bar{M} \otimes q_{1}^{*} \bar{N}_{1} \otimes \cdots \otimes q_{r}^{*} \bar{N}_{r}\right)$ is an isomorphism, where $q_{M}, q_{1}, \ldots, q_{r}$ are the component projections. We will say that $L$ is indecomposable if $(A, L)$ is not the product of polarized abelian varieties of smaller dimension (i.e., the above decomposition only has one factor). In the indecomposable case, note that either $L$ has no fixed component, or $L$ is a principal polarization. We now consider the map $\phi$ associated to $2 L$ for these two cases. Ohbuchi proved the following theorem when $|L|$ has no fixed components.

Theorem 3.2 (Ohbuchi). Suppose $L$ is a polarization of a complex abelian variety $A$ without fixed components, and $\phi$ is the map associated to $2 L$. Then $\phi$ is injective.

Proof. Suppose we have $p, q \in A$ with $\phi(p)=\phi(q)$; i.e., for all $D \in|2 L|, p \in S u p p D$ if and only if $q \in S u p p D$. It is known (see Lemma 4.1.7 on page 72 and Theorem 4.3.6 on page 79 in $\mathrm{Bi}-\mathrm{L}$ ) that there is an irreducible divisor $D_{1} \in|L|$ so that $\tau_{a}^{*} D_{1}=D_{1}$ only for $a=0$ (here $\tau_{a}$ is a translation by $a$ ). Since $L$ has no fixed components, we can choose $D_{2} \in|L|$ irreducible so that $D_{2} \neq(-1)^{*} \tau_{p+q}^{*} D_{1}$. For any $a \in S u p p \tau_{p}^{*} D_{1}, p \in S u p p \tau_{a}^{*} D_{1} \subset \operatorname{Supp}\left(\tau_{a}^{*} D_{1}+\tau_{-a}^{*} D_{2}\right)$. But since $\tau_{a}^{*} D_{1}+$ $\tau_{-a}^{*} D_{2} \in|2 L|$ and $\phi(p)=\phi(q)$ we have $q \in S u p p\left(\tau_{a}^{*} D_{1}+\tau_{-a}^{*} D_{2}\right)$, which implies $a \in \operatorname{Supp}\left(\tau_{q}^{*} D_{1}+(-1)^{*} \tau_{q}^{*} D_{2}\right)$. Therefore,

$$
\operatorname{Supp} \tau_{p}^{*} D_{1} \subset \operatorname{Supp}\left(\tau_{q}^{*} D_{1}+(-1)^{*} \tau_{q}^{*} D_{2}\right) .
$$

Hence,

$$
\operatorname{Supp} D_{1} \subset \operatorname{Supp}\left(\tau_{-p+q}^{*} D_{1}+(-1)^{*} \tau_{p+q}^{*} D_{2}\right) .
$$

Then by choice of $D_{2}$, we have $D_{1}=\tau_{-p+q}^{*} D_{1}$. Hence, $p=q$ by choice of $D_{1}$. 
We now deal with the case where $L$ is an indecomposable principal polarization. When $L$ is a principal polarization, the map associated to $2 L$ maps into $\mathbb{P}^{2 n-1}$ (see Gr-H] ). In this case, we further assume that $L$ is symmetric (see below). Recall that a point $a \in A$ is called a 2-torsion point if $2 a=0$. Notice that if $\alpha: A \rightarrow A$ is the homomorphism $\alpha(x)=2 x$, then the set of 2-torsion points is the kernel of $\alpha$. Hence, the set of 2 -torsion points is isomorphic to $\Lambda / 2 \Lambda \simeq(\mathbb{Z} / 2)^{2 n}$ (i.e., its number is $2^{2 n}$ ). We also need the following concepts.

Definition 3.3. A line bundle $L$ is said to be symmetric if $(-1)^{*} L \simeq L$ (here -1 denotes the map $x \mapsto-x)$. A divisor $D$ is symmetric if $(-1)^{*} D=D$.

Definition 3.4 (Gauss map). Suppose $L$ is a positive line bundle over $A$, and $[L]$ corresponds to a reduced divisor $D$. Since all line bundles over $\mathbb{C}^{n}$ are trivial, the divisor $\pi^{*} D$ is principal, i.e., it is defined by a meromorphic function $\vartheta$. Thus, if $D_{s}$ denotes the smooth part of $S u p p D$ we have a well defined map $G: D_{s} \rightarrow \mathbb{P}^{n-1}$, called the Gauss map, given by $G(p)=\left[\frac{\partial \vartheta}{\partial z_{1}}(p): \cdots: \frac{\partial \vartheta}{\partial z_{n}}(p)\right]$.

It is known that the image of the Gauss map is not contained in a hyperplane (see $\mathrm{Bi}-\mathrm{L}$, page 81 ). The following is stated in $[\mathrm{Bi}-\mathrm{L}$, page 98 . We include a proof here for the sake of completeness.

Theorem 3.5. Suppose that $L$ is an indecomposable symmetric principal polarization of the complex abelian variety $A$, and let $\phi$ be the map determined by $2 L$. Then $d \phi_{p}$ is injective if $p$ is not a 2-torsion point.

Proof. Suppose $p$ is not a 2-torsion point. It suffices to show that given $v \in T_{p}(A)$, there is a divisor $E \in|2 L|$ with $p \in S u p p E$, but $v$ is not tangent to $E$ at $p$. Note that by our assumptions for $L$, there is a unique (necessarily) symmetric $\Theta \in|L|$. Let $G$ denote the Gauss map for $\Theta$. Since $2 p \neq 0$, and the image of $G$ is not contained in a hyperplane, we can choose $q \in S u p p \Theta$ so that $q \notin S u p p \tau_{2 p}^{*} \Theta$, and $v$ is not tangent to $\Theta$ at $q$. Now let $E=\tau_{q-p}^{*} \Theta+\tau_{p-q}^{*} \Theta$. Notice that $E \in|2 L|$. Because $q+(p-q) \in S u p p \tau_{q-p}^{*} \Theta$, we have that $p \in S u p p E$. Since $v$ is not tangent to $\Theta$ at $q$, we know that $v$ is not tangent to $\tau_{q-p}^{*} \Theta$ at $p$. Also, $p \notin S u p p \tau_{p-q}^{*} \Theta$. If it were, then we would have $q \in S u p p \tau_{-2 p}^{*}(-1)^{*} \Theta$. But $(-1)^{*} \Theta=\Theta$, and so $q \in S u p p \tau_{-2 p}^{*} \Theta$, a contradiction. Thus, $v$ is not tangent to $E$ at $p$, as desired.

\section{UNIQUENESS THEOREMS}

To prove our uniqueness results we need the following lemma.

Lemma 4.1. Let $A$ be a complex abelian variety and suppose $f, g: \mathbb{C} \rightarrow A$ are two Zariski dense holomorphic maps. Suppose $\phi: A \rightarrow \mathbb{P}^{N}(\mathbb{C})$ is a holomorphic map, $D$ is a reduced effective ample divisor on $A$, and $H$ is the hyperplane line bundle on $\mathbb{P}^{N}$. Assume that $f(z)=g(z)$ for all $z \in S u p p_{\leq 1} f^{*} D \cup S u p p_{\leq 1} g^{*} D$. If $\mathcal{O}(D) \otimes\left(-2 \phi^{*} H\right)$ is ample, then $\phi \circ f \equiv \phi \circ g$.

Proof. The theorem is trivial if $\phi$ is constant. Suppose $\phi$ is not constant, and by way of contradiction, assume that $\phi \circ f \neq \phi \circ g$. Let $L=\mathcal{O}(D)$. By Theorem 2.2, we have

$$
\begin{aligned}
& \|(1-\epsilon) T_{f}(r, L) \leq N\left(r, f^{*} D_{\leq 1}\right) \\
& \|(1-\epsilon) T_{g}(r, L) \leq N\left(r, g^{*} D_{\leq 1}\right) .
\end{aligned}
$$


Let $S=S u p p_{\leq 1} f^{*} D \cup S u p p_{\leq 1} g^{*} D$, then

$$
\|(1-\epsilon)\left(T_{f}(r, L)+T_{g}(r, L)\right) \leq 2 N(r, S) .
$$

We prove that

$$
N(r, S) \leq T_{f}\left(r, \phi^{*} H\right)+T_{g}\left(r, \phi^{*} H\right)+O(1) .
$$

To do so, define $\Phi: A \times A \rightarrow \mathbb{P}^{N} \times \mathbb{P}^{N}$ by $\Phi\left(a_{1}, a_{2}\right)=\phi\left(a_{1}\right) \times \phi\left(a_{2}\right)$. For $k=1,2$ let $\pi_{k}: \mathbb{P}^{N} \times \mathbb{P}^{N} \rightarrow \mathbb{P}^{N}$, and $p_{k}: A \times A \rightarrow A$ be the canonical projections. Let $H^{\prime}=\pi_{1}^{*} H \otimes \pi_{2}^{*} H$. Then we see that $\Phi^{*} H^{\prime}=\left(\phi \circ p_{1}\right)^{*} H \otimes\left(\phi \circ p_{2}\right)^{*} H$. Let $\Delta$ denote the diagonal of $\mathbb{P}^{N} \times \mathbb{P}^{N}$. We claim that there is a holomorphic section $\rho$ of $H^{\prime}$ so that $\Delta \subset \operatorname{Supp}[\rho]$, but $(\Phi \circ(f \times g))(\mathbb{C})$ is not contained in $\operatorname{Supp}[\rho]$ (here $[\rho]$ denotes the divisor determined by $\rho)$. To see the claim, let $\left(\left[z_{0}, \ldots, z_{N}\right],\left[w_{0}, \cdots, w_{N}\right]\right)$ be homogeneous coordinates for $\mathbb{P}^{N} \times \mathbb{P}^{N}$. Let

$$
P\left(\left[z_{0}, \ldots, z_{N}\right],\left[w_{0}, \cdots, w_{N}\right]\right)=\sum_{0 \leq m<n \leq N} a_{m n}\left(z_{m} w_{n}-z_{n} w_{m}\right),
$$

where the $a_{m n}$ are complex numbers not all zero. $P$ determines a holomorphic section $\rho$ of $H^{\prime}$. Evidently, $\Delta \subset S u p p[\rho]$. By way of contradiction, suppose for all choices of $a_{m n}$ we have that the image of $\Phi \circ(f \times g)$ is contained in $\operatorname{Supp}[\rho]$. In this case, we must have $P \circ \Phi \circ(f \times g) \equiv 0$. Write $\phi \circ f=\left[F_{0}, \cdots, F_{N}\right]$ and $\phi \circ g=\left[G_{0}, \cdots, G_{N}\right]$. Then,

$$
\sum_{0 \leq m<n \leq N} a_{m n}\left[\left(F_{m} G_{n}\right)-\left(F_{n} G_{m}\right)\right] \equiv 0
$$

for all choices $a_{m n}$. Thus, we must have for all $0 \leq m<n \leq N$

$$
F_{m} G_{n}-F_{n} G_{m} \equiv 0 .
$$

But then, $\phi \circ f=\phi \circ g$, which is a contradiction, and so the claim is proved. Now, choose $\rho$ as in the claim. Since $f \equiv g$ on $S$, we have that $(\Phi \circ(f \times g))(S) \subset \Delta \subset$ $\operatorname{Supp}[\rho]$, and we have that the image of $\Phi \circ(f \times g)$ is not contained in $\operatorname{Supp}[\rho]$. Therefore,

$$
N(r, S) \leq N\left(r,(f \times g)^{*}[\rho \circ \Phi]\right) .
$$

Now, $\rho \circ \Phi$ is a holomorphic section of $\Phi^{*} H^{\prime}$, and so $N\left(r,(f \times g)^{*}[\rho \circ \Phi]\right) \leq$ $T_{f \times g}\left(r, \Phi^{*} H^{\prime}\right)+O(1)$. We know that

$T_{f \times g}\left(r, \Phi^{*} H^{\prime}\right)=T_{f \times g}\left(r,\left(\phi \circ p_{1}\right)^{*} H \otimes\left(\phi \circ p_{2}\right)^{*} H\right) \leq T_{f}\left(r, \phi^{*} H\right)+T_{g}\left(r, \phi^{*} H\right)+O(1)$. So, $N(r, S) \leq T_{f}\left(r, \phi^{*} H\right)+T_{g}\left(r, \phi^{*} H\right)+O(1)$ as desired. Hence, we get

$$
(1-\epsilon)\left(T_{f}(r, L)+T_{g}(r, L)\right) \leq 2 T_{f}\left(r, \phi^{*} H\right)+2 T_{g}\left(r, \phi^{*} H\right)+O(1) .
$$

Let $L_{0}=L \otimes\left(-2 \phi^{*} H\right)$, then

$$
T_{f}\left(r, L_{0}\right)+T_{g}\left(r, L_{0}\right) \leq \epsilon\left(T_{f}(r, L)+T_{g}(r, L)\right) .
$$

By assumption, $L_{0}$ is ample, so there exist $\mu>0$ such that $\left(\mu L_{0}\right) \otimes(-L)$ is big. Hence,

$$
T_{f}(r, L)+T_{g}(r, L) \leq \mu\left(T_{f}\left(r, L_{0}\right)+T_{g}\left(r, L_{0}\right)\right) .
$$

Therefore,

$$
\| T_{f}\left(r, L_{0}\right)+T_{g}\left(r, L_{0}\right) \leq \mu \epsilon\left(T_{f}\left(r, L_{0}\right)+T_{g}\left(r, L_{0}\right)\right),
$$

which gives a contradiction. Therefore, $\phi \circ f \equiv \phi \circ g$.

The main result of this paper is the following theorem. 
Main Theorem. Let $A$ be a complex abelian variety, $\operatorname{dim} A=n$, and let $D$ be a reduced effective divisor with $D=D_{1}+\cdots+D_{q}$ and $D_{i}$ irreducible for all $i$. Assume that

(1) $\mathcal{O}\left(D_{1}\right)$ is ample and symmetric.

(2) $\mathcal{O}\left(D_{2}\right) \otimes \cdots \otimes \mathcal{O}\left(D_{q}\right) \otimes \mathcal{O}\left(-3 D_{1}\right)$ is ample.

(3) For at least one $i, S u p p D_{i}$ contains no 2-torsion points.

If $f, g: \mathbb{C} \rightarrow A$ are Zariski dense holomorphic maps, and if $f(z)=g(z)$ for all $z \in \operatorname{Supp}_{\leq 1} f^{*} D \cup \operatorname{Supp}_{\leq 1} g^{*} D$, then $f \equiv g$.

Proof. Let $\phi: A \rightarrow \mathbb{P}^{N}$ denote the map associated to $\mathcal{O}\left(2 D_{1}\right)$. Let $H$ denote the hyperplane line bundle on $\mathbb{P}^{N}$, then we have that $\phi^{*} H=\mathcal{O}\left(2 D_{1}\right)$. Therefore, $\mathcal{O}(D) \otimes\left(-2 \phi^{*} H\right)=\mathcal{O}\left(D_{2}\right) \otimes \cdots \otimes \mathcal{O}\left(D_{q}\right) \otimes \mathcal{O}\left(-3 D_{1}\right)$. Under assumption (2), $\mathcal{O}(D) \otimes\left(-2 \phi^{*} H\right)>0$. So, Lemma 4.1 implies that $\phi \circ f=\phi \circ g$. Now, the irreducibility implies that the fixed component of $\left|D_{1}\right|$ is either empty or equal to $D_{1}$. If the fixed component of $\left|D_{1}\right|$ is empty, then, by Theorem 3.2, $\phi$ is injective. This implies that $f \equiv g$. If the fixed component of $\left|D_{1}\right|$ is $D_{1}$, then $E \geq D_{1}$ for all $E \in\left|D_{1}\right|$. Write $E=D_{1}+(h)$. Then, $(h) \geq 0$ which implies that $h$ must be constant since $A$ is compact. So we get $E=D_{1}$. This means that $\operatorname{dim} H^{0}\left(A, \mathcal{O}\left(D_{1}\right)\right)=1$. Hence $\mathcal{O}\left(D_{1}\right)$ is principally polarized. Moreover, the irreducibility of $D_{1}$ implies that when one writes the decomposition $F_{1}+\cdots+F_{r}$ of the fixed component (which is $\left.D_{1}\right)$, we have $r=1$. So $\mathcal{O}\left(D_{1}\right)$ is an indecomposable principal polarization of $A$. By the assumption, $\mathcal{O}\left(D_{1}\right)$ is also symmetric. Theorem 3.5 then implies that $\phi$ is locally injective outside of the 2 -torsion points. By the assumption, we may choose $i \in\{1, \ldots, q\}$ so that $S u p p D_{i}$ contains no 2-torsion points of $A$. Since $f$ and $g$ are Zariski dense, by Theorem 2.2, both $S u p p_{\leq 1} f^{*} D_{i}$ and $S u p p_{\leq 1} g^{*} D_{i}$ are non-empty. Moreover, since $f=g$ on $\operatorname{Supp}_{\leq 1} f^{*} D \cup S u p p_{\leq 1} g^{*} D$, there is $z_{0} \in \mathbb{C}$ such that $f\left(z_{0}\right)=g\left(z_{0}\right) \in S u p p D_{i}$. By assumption (3) we know that $f\left(z_{0}\right)$ is not a 2-torsion point on $A$. Theorem 3.5 implies that there is a neighborhood of $f\left(z_{0}\right)$ on which $\phi$ is injective. Thus, there is a neighborhood $U$ of $z_{0}$ so that $f=g$ on $U$. But then, $f \equiv g$ by uniqueness of analytic continuation.

Remark. From the proof we see that if $\mathcal{O}\left(D_{1}\right)$ has no fixed components, then we actually do not need the "symmetric" assumption.

Corollary 4.2. Suppose $A$ is a complex abelian variety, and $D=D_{1}+\cdots+D_{5}$ a reduced effective divisor with $D_{i}$ irreducible so that

(1) $L:=\mathcal{O}\left(D_{1}\right)$ is ample and symmetric.

(2) $c_{1}\left(\mathcal{O}\left(D_{1}\right)\right)=\cdots=c_{1}\left(\mathcal{O}\left(D_{5}\right)\right)$.

(3) For at least one $i, S u p p D_{i}$ contains no 2-torsion points.

If $f, g: \mathbb{C} \rightarrow A$ are Zariski dense holomorphic maps, and if $f(z)=g(z)$ for all $z \in \operatorname{Supp}_{\leq 1} f^{*} D \cup \operatorname{Supp}_{\leq 1} g^{*} D$, then $f \equiv g$.

Proof. By assumption $(2) c_{1}(\mathcal{O}(D))-4 c_{1}(L)=5 c_{1}(L)-4 c_{1}(L)=c_{1}(L)>0$. Thus, condition (2) of the Main Theorem is satisfied, and so $f \equiv g$.

Notice that when $n=1$, this gives an improvement of Schmid's result for elliptic curves.

Corollary 4.3. Let $A$ be an elliptic curve and let $a_{1}, \cdots, a_{5}$ be distinct points of $A$. If $f, g: \mathbb{C} \rightarrow A$ are non-constant holomorphic, and assume that for all $z \in \bigcup_{j=1}^{5}\left(\operatorname{Supp}_{\leq 1} f^{*}\left(a_{j}\right) \cup \operatorname{Supp}_{\leq 1} g^{*}\left(a_{j}\right)\right)$, we have $f(z)=g(z)$. Then $f \equiv g$. 
Proof. In the one-dimensional case we may identify $H^{2}(A, \mathbb{Z})$ with $\mathbb{Z}$. Set $D=$ $D_{1}+\cdots+D_{5}$ where $D_{i}=\left(a_{i}\right)$. Let $L_{i}$ be the line bundle determined by $D_{i}$. Notice that $c_{1}\left(L_{i}\right)=1$ for all $i$. Also, by a translation, we can assume that $a_{1}=O$, so $L_{1}$ is a indecomposable symmetric principal polarization. Since $\operatorname{dim} A=1, A$ has exactly four 2 -torsion points. So, at least one $a_{i}$ is not a 2 -torsion point. Thus, by the preceding corollary $f \equiv g$.

Finally, in the one-dimensional case, the number of 2-torsions is 4 , so if our divisor has 5 components (points), then at least one of these points has a neighborhood where $\phi$ is injective. However, if $A$ has dimension larger than 1, the number of 2 -torsions is $2^{2 \operatorname{dim} A}>5$. So, to obtain the uniqueness using only 5 components, the price we paid is the addition of the hypothesis that one component of the divisor contains no 2-torsion point. However, recall that $3 L$ is very ample for any polarization $L$ of $A$. Using this, we can eliminate some of our assumptions by increasing the number of components. More precisely, we have the following results.

Theorem 4.4. Let $A$ be a complex abelian variety. Suppose $D=D_{1}+\cdots+D_{k}$ is a reduced effective divisor with $D_{i}$ irreducible. Suppose $\mathcal{O}\left(D_{1}\right)$ is ample and $\mathcal{O}\left(D_{2}\right) \otimes \cdots \otimes \mathcal{O}\left(D_{k}\right) \otimes \mathcal{O}\left(-5 D_{1}\right)$ is ample. Let $f, g: \mathbb{C} \rightarrow A$ be two Zariski dense holomorphic maps, and if $f(z)=g(z)$ for all $z \in S u p p_{\leq 1} f^{*} D \cup S u p p_{\leq 1} g^{*} D$, then $f \equiv g$.

Proof. Since $D_{1}$ is ample, $L:=\mathcal{O}\left(D_{1}\right)>0$ and hence, the map $\phi$ associated to $3 L$ is an embedding into $\mathbb{P}^{N}$ for some $N$. If $H$ is the hyperplane bundle over $\mathbb{P}^{N}$, then $\phi^{*} H=3 L$. By hypothesis, $c_{1}(\mathcal{O}(D))-6 c_{1}(L)>0$ hence, $\mathcal{O}(D) \otimes\left(-2 \phi^{*} H\right)>0$. So by Lemma 4.1, we must have $\phi \circ f=\phi \circ g$. But $\phi$ is injective hence, $f \equiv g$.

Corollary 4.5. Suppose $A$ is a complex abelian variety, $D=D_{1}+\cdots+D_{7}$ is a reduced effective divisor where $D_{1}$ is ample, all $D_{i}$ are irreducible and $c_{1}\left(\mathcal{O}\left(D_{1}\right)\right)=$ $\cdots=c_{1}\left(\mathcal{O}\left(D_{7}\right)\right)$. If $f, g: \mathbb{C} \rightarrow A$ are Zariski dense holomorphic maps, and if $f(z)=g(z)$ for all $z \in \operatorname{Supp}_{\leq 1} f^{*} D \cup \operatorname{Supp}_{\leq 1} g^{*} D$, then $f \equiv g$.

\section{Further eXtension of Schmid's Results}

In this section, we consider the case of $l \geq 2$ non-constant, holomorphic curves into an elliptic curve. If $E$ is an elliptic curve, and $l \geq 2$, we let $E^{l}$ be the product of $l$ copies of $E$. Define $\Gamma \subset E^{l}$, by $\Gamma=\bigcup_{1<i<j \leq l}\left\{\left(a_{1}, \ldots, a_{l}\right) \mid a_{i}=a_{j}\right\}$. Note that in the case $l=2, \Gamma$ is just the diagonal, $\Delta$. To prove our result, we need the following analogue of Lemma 4.1 above.

Lemma 5.1. Suppose $E$ is an elliptic curve, $l \geq 2$ an integer, and $f_{1}, \ldots, f_{l}: \mathbb{C} \rightarrow$ $E$ are non-constant, holomorphic maps. Suppose $\phi: E \rightarrow \mathbb{P}^{1}$ is holomorphic, $D$ is an effective reduced ample divisor on $E$, and $H$ is the hyperplane line bundle on $\mathbb{P}^{1}$. Let $S=\bigcup_{j=1}^{l} S_{\text {upp }} f_{j}^{*} D$. Assume that $\left(f_{1} \times \cdots \times f_{l}\right)(S) \subset \Gamma$, and $\mathcal{O}(D) \otimes\left(-l \phi^{*} H\right)>0$, then for some $i \neq j$, we have $\phi \circ f_{i} \equiv \phi \circ f_{j}$.

Proof. This is trivial if $\phi$ is constant. So, suppose $\phi$ is not constant, and for each $j$, let $F_{j}=\phi \circ f_{j}$. Assume that for all $i \neq j$ that $F_{i} \not \equiv F_{j}$. Write $L=\mathcal{O}(D)$; then by Theorem 2.2 we have for each $j \in\{1, \cdots, l\}$

$$
\|(1-\epsilon) T_{f_{j}}(r, L) \leq N\left(r, f_{j}^{*} D_{\leq 1}\right) .
$$


Thus,

$$
\|(1-\epsilon) \sum_{j=1}^{l} T_{f_{j}}(r, L) \leq l N(r, S) .
$$

Similar to the proof of Lemma 4.1, we claim that

$$
N(r, S) \leq \sum_{j=1}^{l} T_{f_{j}}\left(r, \phi^{*} H\right)+O(1) .
$$

Define $\Phi: E^{l} \rightarrow \mathbb{P}^{l}$ via $\Phi\left(a_{1}, \ldots, a_{l}\right)=\phi\left(a_{1}\right) \times \cdots \times \phi\left(a_{l}\right)$. For each $k$, let $\pi_{k}:$ $\mathbb{P}^{1} \rightarrow \mathbb{P}^{1}$, and $p_{k}: E^{l} \rightarrow E$ be the canonical projections. Define $H^{\prime}=\bigotimes_{k=1}^{l} \pi_{k}^{*} H$; then we see that $\Phi^{*} H^{\prime}=\bigotimes_{k=1}^{l}\left(\phi \circ p_{k}\right)^{*} H$. For each $1 \leq j \leq l$, let $z^{(j)}=\left[z_{0}^{(j)}\right.$ : $z_{1}^{(j)}$ ] be homogeneous coordinates on the $j$-th copy of $\mathbb{P}^{1}$, and define $\Gamma^{\prime} \subset \mathbb{P}^{1}$ by $\Gamma^{\prime}=\bigcup_{1 \leq i<j \leq l}\left\{\left(z^{(1)}, \ldots, z^{(l)}\right) \mid z^{(i)}=z^{(j)}\right\}$. Define a polynomial

$$
P\left(z^{(1)}, \ldots, z^{(l)}\right)=\prod_{1 \leq i<j \leq l}\left(z_{0}^{(i)} z_{1}^{(j)}-z_{1}^{(i)} z_{0}^{(j)}\right) .
$$

This polynomial defines in a natural way a section $\rho$ of $H^{\prime}$. Evidently, $\Gamma^{\prime} \subset \operatorname{Supp}[\rho]$. Also notice that $\left(\Phi \circ\left(f_{1} \times \cdots \times f_{l}\right)\right)(\mathbb{C})=\left(F_{1} \times \cdots \times F_{l}\right)(\mathbb{C})$ is not contained in $\operatorname{Supp}[\rho]$. To see this, suppose not and write $F_{j}=\left[F_{0}^{j}: F_{1}^{j}\right]$. Then we have

$$
\prod_{1 \leq i<j \leq l}\left(F_{0}^{i} F_{1}^{j}-F_{1}^{i} F_{0}^{j}\right) \equiv 0 .
$$

But then, at least one factor, $F_{0}^{i} F_{1}^{j}-F_{1}^{i} F_{0}^{j}$ must vanish on some $B \subset \mathbb{C}$ which has an accumulation point (if not, then the product vanishes only on a discrete subset of $\mathbb{C}$ ). Hence, $F_{0}^{i} F_{1}^{j}-F_{1}^{i} F_{0}^{j} \equiv 0$. That is, $F_{i}=F_{j}$, with $i<j$, which contradicts our assumption, and proves the assertion. Now, $\left(f_{1} \times \cdots \times f_{l}\right)(S) \subset \Gamma$, hence $\left(F_{1} \times \cdots \times F_{l}\right)(S) \subset \Gamma^{\prime} \subset S u p p[\rho]$. Proceeding as in the proof of Lemma 4.1, we conclude that $N(r, S) \leq \sum_{j=1}^{l} T_{f_{j}}\left(r, \phi^{*} H\right)+O(1)$, which proves the claim. Setting $L_{0}=L \otimes\left(-l \phi^{*} H\right)$, the argument from Lemma 4.1 implies that for some $\mu>0$ we have

$$
\| \sum_{j=1}^{l} T_{f_{j}}(r, L) \leq \mu \epsilon \sum_{j=1}^{l} T_{f_{j}}(r, L),
$$

a contradiction. Thus, for some $i \neq j$, we have $\phi \circ f_{i}=\phi \circ f_{j}$, as desired.

Using this we can derive the following uniqueness result which gives an extension of Schmid's result.

Theorem 5.2. Suppose $E$ is an elliptic curve, $l \geq 2$ is an integer, and that $a_{1}, \cdots, a_{2 l+1}$ are distinct points of $E$. Suppose that $f_{1}, \cdots, f_{l}: \mathbb{C} \rightarrow E$ are nonconstant, holomorphic maps, and that for all $z \in \bigcup_{j=1}^{2 l+1}\left(\operatorname{Supp}_{\leq 1} f_{1}^{*}\left(a_{j}\right) \cup \cdots \cup\right.$ $\left.\operatorname{Supp}_{\leq 1} f_{l}^{*}\left(a_{j}\right)\right)$ there exists $1 \leq i_{1} \neq i_{2} \leq l$ (which may depend on $z$ ) such that $f_{i_{1}}(z)=f_{i_{2}}(z)$. Then $f_{i} \equiv f_{j}$ for some $i \neq j$.

Proof. Let $D$ be the divisor $D=\left(a_{1}\right)+\cdots+\left(a_{2 l+1}\right)$. By a translation, suppose that $a_{1}=O$. Since $L_{1}=\mathcal{O}\left(a_{1}\right)$ is a symmetric, principal polarization, the map $\phi$ associated to $2 L_{1}$ maps into $\mathbb{P}^{1}$, and is locally injective outside the four 2-torsion points of $E$. Also notice that, $\mathcal{O}(D)-\left(l \phi^{*} H\right) \simeq(2 l+1) L_{1}-2 l L_{1}=L_{1}>0$, and, by the assumption, for all $z \in \bigcup_{j=1}^{2 l+1}\left(\operatorname{Supp}_{\leq 1} f_{1}^{*}\left(a_{j}\right) \cup \cdots \cup S u p p_{\leq 1} f_{l}^{*}\left(a_{j}\right)\right)$, we have 
$\left(f_{1} \times \cdots \times f_{l}\right)(z) \in \Gamma$. So, by Lemma 5.1, we know that $\phi \circ f_{i}=\phi \circ f_{j}$ for some $i \neq j$. Since $2 l+1>4$, there is a $k$ so that $\phi$ is injective in a neighborhood of $a_{k}$. Since $f_{i}$ omits no point of $E$, this implies that there is a neighborhood $U$ of some $z_{0} \in \mathbb{C}$ so that $f_{i}=f_{j}$ on $U$, hence $f_{i} \equiv f_{j}$.

\section{Algebraic dependence}

In this section, we study the algebraic dependence for a set of Zariski dense holomorphic mappings into an abelian variety. Let $f_{1}, \cdots, f_{l}: \mathbb{C} \rightarrow A$ be holomorphic maps. They are said to be algebraically dependent over $\mathbb{C}$ if there exists some nondecomposable hypersurface $\Sigma$ in $A^{l}=A \times \cdots \times A$ such that $\left(f_{1} \times \cdots \times f_{l}\right)(\mathbb{C}) \subset \Sigma$; here decomposable means there are integers $l_{1}, \ldots, l_{s}$ with $l=l_{1}+\cdots+l_{s}$ and algebraic sets $\Sigma_{j} \subset A^{l_{j}}$ and $\Sigma=\Sigma_{1} \times \ldots \times \Sigma_{s}$. To be more precise, we make the following definition.

Definition 6.1. Let $l>0$ be an integer, $S \subset \mathbb{C}$, and $f_{1}, \cdots, f_{l}: \mathbb{C} \rightarrow A$ be holomorphic. If there is a proper non-decomposable algebraic subset $\Sigma \subset A^{l}$ so that $\left(f_{1} \times \cdots \times f_{l}\right)(S) \subset \Sigma$, then we say that $f_{1}, \ldots, f_{l}$ are $\Sigma$-related on $S$.

To state our result, we first fix some notation. An element of $\operatorname{Pic}(A) \otimes \mathbb{Q}$ is called a $\mathbb{Q}$-line bundle over $A$. A $\mathbb{Q}$-line bundle $F$ is said to be big (or ample) if there is a $\nu \in \mathbb{N}$ so that $\nu F \in \operatorname{Pic}(A)$ is big (or ample). Further, we define $T_{f}(r, F)=\frac{1}{\nu} T_{f}(r, \nu F)$. Evidently, this is well defined. Choose big line bundles $F_{1}, \ldots, F_{l}$ over $A$. Define a line bundle on $A^{l}$ by $\tilde{F}=p_{1}^{*} F_{1} \otimes \cdots \otimes p_{l}^{*} F_{l}$, where $p_{1}, \ldots, p_{l}: A^{l} \rightarrow A$ are component projections. Let $\Sigma$ be a given non-decomposable hypersurface and let $\tilde{L}$ be a big line bundle over $A^{l}$ such that $\Sigma=S u p p \tilde{D}$ for some $\tilde{D} \in|\tilde{L}|$. We assume that there exists a positive rational number $\tilde{\gamma}$ such that

$$
(\tilde{\gamma} \tilde{F}) \otimes(-\tilde{L}) \text { is big. }
$$

Assume that there is a line bundle $F_{0}$ with the property that $F_{0} \otimes\left(-F_{j}\right) \geq 0$ for all $j$ (if $F_{1}=\cdots=F_{l}$, then we can just take $F_{0}:=F_{1}$ ).

We have the following theorem (compare to Theorem 2.2 in [Ai2]).

Theorem 6.2. With the above notation and assumptions, let $f_{1}, \cdots, f_{l}: \mathbb{C} \rightarrow A$ be Zariski dense holomorphic maps. Let $D$ be an effective reduced ample divisor on A (not necessarily irreducible). Assume that

$$
\mathcal{O}(D) \otimes\left(-l \tilde{\gamma} F_{0}\right) \text { is big. }
$$

Let $S=\bigcup_{i=1}^{l} S u p p_{\leq 1} f_{i}^{*} D$ and assume that $f_{1}, \ldots, f_{l}$ are $\Sigma$-related on $S$. Then $f_{1}, \ldots, f_{l}$ are $\Sigma$-related on $\mathbb{C}$.

Proof. Write $\tilde{f}=f_{1} \times \cdots \times f_{l}$. Suppose that $\tilde{f}(\mathbb{C}) \not \subset \Sigma$. From Theorem 2.2, we have, for every $1 \leq i \leq l$,

$$
\|(1-\epsilon) T_{f_{i}}(r, \mathcal{O}(D)) \leq N\left(r, f_{i}^{*} D_{\leq 1}\right) .
$$

Hence,

$$
\| \sum_{i=1}^{l}(1-\epsilon) T_{f_{i}}(r, \mathcal{O}(D)) \leq l N(r, S) .
$$


From the hypothesis,

$$
\begin{aligned}
N(r, S) & \leq N\left(r, \tilde{f}^{*} \tilde{D}\right) \\
& \leq T_{\tilde{f}}(r, \tilde{L})+O(1) \\
& \leq \tilde{\gamma} T_{\tilde{f}}(r, \tilde{F})+O(1) \\
& \leq \tilde{\gamma} \sum_{i=1}^{l} T_{f_{i}}\left(r, F_{i}\right) \\
& \leq \tilde{\gamma} \sum_{i=1}^{l} T_{f_{i}}\left(r, F_{0}\right) .
\end{aligned}
$$

Combining these facts we get the inequality

$$
\| \sum_{i=1}^{l}(1-\epsilon) T_{f_{i}}(r, \mathcal{O}(D)) \leq l \tilde{\gamma} \sum_{i=1}^{l} T_{f_{i}}\left(r, F_{0}\right) .
$$

Let

$$
L_{0}=\mathcal{O}(D) \otimes\left(-l \tilde{\gamma} F_{0}\right)
$$

then,

$$
\| \sum_{i=1}^{l} T_{f_{i}}\left(r, L_{0}\right) \leq \epsilon \sum_{i=1}^{l} T_{f_{i}}(r, \mathcal{O}(D)) .
$$

Since, by the assumption, $L_{0}$ is big, there exists $\mu>0$ such that $\mu L_{0} \otimes(-\mathcal{O}(D))$ is big. Thus,

$$
T_{f_{i}}(r, \mathcal{O}(D)) \leq \mu T_{f_{i}}\left(r, L_{0}\right)
$$

Therefore,

$$
\| \sum_{i=1}^{l} T_{f_{i}}\left(r, L_{0}\right) \leq \mu \epsilon \sum_{i=1}^{l} T_{f_{i}}\left(r, L_{0}\right),
$$

which gives a contradiction. Thus, $\tilde{f}(\mathbb{C}) \subset \Sigma$ as desired.

We obtain the following uniqueness result as an application of this theorem.

Corollary 6.3. Suppose that $D=D_{1}+\cdots+D_{q}$ is a reduced effective divisor on $A$ with all $D_{i}$ ample, irreducible, and $c_{1}\left(\mathcal{O}\left(D_{1}\right)\right)=\cdots=c_{1}\left(\mathcal{O}\left(D_{q}\right)\right)$. Let $\Delta$ be the diagonal of $A \times A$, and let $\tilde{L}$ be the line bundle determined by the diagonal divisor $\Delta$. Assume that there exists a positive rational number $\tilde{\gamma}$ such that $\left(\tilde{\gamma}\left(p_{1}^{*} \mathcal{O}\left(D_{1}\right) \otimes\right.\right.$ $\left.\left.p_{2}^{*} \mathcal{O}\left(D_{1}\right)\right)\right) \otimes(-\tilde{L})$ is ample. If $f, g: \mathbb{C} \rightarrow A$ are Zariski dense holomorphic maps so that $f(z)=g(z)$ on $\operatorname{Supp}_{\leq 1} f^{*} D \cup \operatorname{Supp}_{\leq 1} g^{*} D$, and $q>2 \tilde{\gamma}$, then $f \equiv g$.

Proof. Using our above notation, we set $F_{0}=F_{1}=F_{2}=\mathcal{O}\left(D_{1}\right)$, and so $\tilde{F}=$ $p_{1}^{*} \mathcal{O}\left(D_{1}\right) \otimes p_{2}^{*} \mathcal{O}\left(D_{1}\right)$. Since $q>2 \tilde{\gamma}$, and $c_{1}\left(\mathcal{O}\left(D_{1}\right)\right)=\cdots=c_{1}\left(\mathcal{O}\left(D_{q}\right)\right)$, we have

$$
c_{1}(\mathcal{O}(D))-2 \tilde{\gamma} c_{1}\left(F_{0}\right)=(q-2 \tilde{\gamma}) c_{1}\left(\mathcal{O}\left(D_{1}\right)\right)>0 .
$$

By Theorem $6.2, f$ and $g$ are $\Delta$-related on $\mathbb{C}$, that is $f \equiv g$.

In the case of elliptic curve that $A=E$, Katsura (see Ai2]) proved that, if $D_{1}=(p)$, where $p \in E$, then $\left(2\left(p_{1}^{*} \mathcal{O}\left(D_{1}\right) \otimes p_{2}^{*} \mathcal{O}\left(D_{1}\right)\right)\right) \otimes(-\tilde{L})$ is ample (i.e., we can take $\tilde{\gamma}=2)$. Hence, if we let $D=\left(a_{1}\right)+\cdots+\left(a_{q}\right)$ where $a_{1}, \ldots, a_{q}$ are distinct points in $E$, assume that for every $z \in \bigcup_{j=1}^{q}\left(S u p p_{\leq 1} f^{*} a_{j} \cup S u p p_{\leq 1} g^{*} a_{j}\right), f(z)=g(z)$, 
then the conditions in Corollary 6.3 are satisfied for $q>4$. This implies that $f \equiv g$ for $q>4$, which recovers Schmid's theorem.

\section{REFERENCES}

[Ai1] Aihara, Y. A Unicity Theorem for Meromorphic Mappings into Compactified Locally Symmetric Spaces. Kodai Math J. 14 (1991) no. 3, 392-405. MR.1131922 (92j:32097)

[Ai2] Aihara, Y. Algebraic Dependence of Meromorphic Mappings in Value Distribution Theory. Nagoya Math. J. 169 (2003) 145-178. MR.1962526 (2004g:32015)

[Bi-L] Birkenhake, C. and Lange, H. Complex Abelian Varieties. Springer (2003). MR2062673 (2005c:14001)

[Dr] Drouilhet, S.J. A Unicity Theorem for Meromorphic Maps Between Algebraic Varieties. Trans. Amer. Math Soc. 265 (1981) no. 2, 349-358. MR610953 (82m:32021)

[Fu] Fujimoto, H. Uniqueness Problem with Truncated Multiplicities in Value Distribution Theory. Nagoya Math J. 152 (1998) 131-152. MR1659377|(99m:32029)

[Gr-H] Griffiths, Ph. and Harris, J. Principles of Algebraic Geometry. John Wiley and Sons (1997). MR.1288523 (95d:14001)

[Ko-O] Kobayashi, S. and Ochiai, T. Mappings into Compact Complex Manifolds with Negative First Chern Class. J. Math Soc. Japan 23 (1971) 137-148. MR0288316 (44:5514)

[No-W-Y] Noguchi, J., Winklemann, J. and Yamanoi, K. The Second Main Theorem for Holomorphic Curves into Semi-Abelian Varieties II. Forum Math. 20 (2008) no. 3, 469-503. MR2418202 (2009f:32031)

[Ohb] Ohbuchi, A. Some Remarks on Ample Line Bundles of Abelian Varieties. Manuscripta Math. 57 (1987) no. 2, 225-238. MR871633 (87m:14051)

[Sc] Schmid, E.M. Some Theorems of Value Distributions of Meromorphic Functions. Math Z. 120 (1971) 61-92. MR0284583(44:1808)

[Si-Y] Siu, Y.T.and Yeung, S.K. Generalized Bloch's Theorem and the Hyperbolicity of the Complement of an Ample Divisor in an Abelian Variety. Math Ann. 306 (1996) no. 4, 743-758. MR 1418351 (97g:32028)

[Ya] Yamanoi, K. Holomorphic Curves in Abelian Varieties and Intersections with Higher Codimensional Subvarieties. Forum Math 16 (2004) no. 5, 749-788. MR2096686 $(2005 \mathrm{j}: 32017)$

Department of Mathematics, University of Houston, Houston, Texas 77204

E-mail address: dulock11@math.uh.edu

Current address: Department of Mathematics, University of North Texas, Denton, Texas 76203-5017

Department of Mathematics, University of Houston, Houston, Texas 77204

E-mail address: minru@math.uh.edu 